\title{
THE POINCARÉ CONJECTURE IS TRUE IN THE PRODUCT OF ANY GRAPH WITH A DISK
}

\author{
DAVID GILLMAN
}

(Communicated by Frederick R. Cohen)

\begin{abstract}
We prove that the only compact 3-manifold-with-boundary which has trivial rational homology, and which embeds in the product of a graph with a disk, is the 3-ball. This implies that no punctured lens space embeds in the product of a graph with a disk. It also implies our title.

The proof relies on a general position argument which enables us to perform surgery.
\end{abstract}

\section{INTRODUCTION AND SUMMARY}

We work with finite polyhedra and piecewise linear maps throughout. The term 3-manifold $M$ denotes a compact, but not necessarily connected, 3manifold-with-boundary. We denote by $b_{i}(M)$ the $i$ th betti number of $M$; that is, the rank of the $i$ th homology group of $M$ with rational coefficients; thus, $b_{0}(M)=1$ if and only if $M$ is connected.

Definition I. Let $n$ be a nonnegative integer. The n-punctured ball is the topologically unique 3-manifold obtained by removing $n$ disjoint open neighborhoods of $n$ interior points from the 3-dimensional ball.

Theorem. Let $G$ denote a graph (that is, a 1-complex), and I denote an interval. Let $M$ be a 3-manifold embedded in $G \times I \times I$. If $b_{0}(M)=1$ and $b_{1}(M)=0$, then $M$ is homeomorphic to the n-punctured ball with $n=b_{2}(M)$.

In other words, any 3-manifold $M$ in $G \times I \times I$ with $b_{0}(M)=1$ and $b_{1}(M)=0$ is completely classified by its three betti numbers $b_{0}, b_{1}$, and $b_{2}$.

Corollary I. Let $M$ denote any connected 3-manifold with trivial raitonal homology. If $M$ embeds in $G \times I \times I$, then $M$ is the 3-ball.

Corollary 2. The punctured lens spaces do not embed in $G \times I \times I$. The product $P^{2} \times I$ of a projective plane and an interval does not embed in $G \times I \times I$. Furthermore, these 3-manifolds, punctured any finite number of times, fail to embed in $G \times I \times I$.

Received by the editors March 3, 1989 and, in revised form, November 7, 1989.

1980 Mathematics Subject Classification (1985 Revision). Primary 57M40; Secondary 57D65. 
This fact about the punctured lens spaces does not follow the odd-even pattern of known results about embeddings in Euclidean 4-space. The odd punctured lens spaces embed in 4-space [5]. The even ones do not (differentiably) embed in 4-space [1].

Corollary 3. The Poincare Conjecture holds in $G \times I \times I$; that is, the only contractible 3-manifold in $G \times I \times I$ is the 3-ball.

Question. Does the Theorem hold in the product $K^{2} \times I$ of any contractible 2-complex $K^{2}$ and an interval?

An affirmative answer to this question implies the Poincare Conjecture, because: Let $K_{\text {std }}$ denote any standard spine of a contractible 3-manifold $M$. It is shown in [2] that $M$ embeds in $K_{\text {std }} \times I$.

Indeed, we do not know the answer to this question for the special case, where $K^{2}$ is the cone over a graph.

\section{Alternative STATEMENTS OF THE THEOREM}

Let us call the statement given above Version I of the Theorem. To begin, we verify that Version $I$ is valid in 3-space.

Lemma I. Any 3-manifold $M$ in $I \times I \times I$ with $b_{0}(M)=1$ and $b_{1}(M)=0$ is an $n$-punctured ball with $n=b_{2}(M)$.

Proof. Because $M$ lies in $I \times I \times I, M$ is orientable. Since $b_{1}(M)=0$ and $M$ is orientable, $b d M$ is a finite collection of 2-spheres [4, p. 231]. An induction argument is now used on the number of 2-spheres. The 3-dimensional Schoenflies theorem [3, Ch. 17] is applied on the innermost 2-sphere to complete the proof of Lemma 1.

If one removes the restriction that $b_{0}(M)=1$, a counterexample to classification of manifolds in 3-space by betti numbers is easily constructed: Let $b_{0}(M)=2, b_{1}(M)=0$, and $b_{2}(M)=2$. Two distinct submanifolds of 3space have these betti numbers. Both manifolds consist of two disjoint 3-balls with two punctures. One may place both punctures in the same 3-ball, or one in each 3-ball.

If one instead removes the restriction that $b_{1}(M)=0$ from Lemma 1 , then a solid torus and a cube-with-a-knotted-hole provide the counterexample to classification by betti numbers.

Version II of the theorem. Any 3-manifold $M$ in $G \times I \times I$ with $b_{1}(M)=0$ is embeddable in $I \times I \times I$.

Without the hypothesis that $b_{1}(M)=0$, Version II is false. The 3-manifold Punc $S^{2} \times S^{1}$, obtained by puncturing $S^{2} \times S^{1}$ exactly once, is a counterexample. Specifically, Punc $S^{2} \times S^{1}$ embeds in $T \times I \times I$, where $T$ denotes a triod. To see this, first place a solid torus in 3-space. Observe that a meridianal annulus on its boundary is planar in 3-space. Onto this planar annulus, we now attach a 2-handle which lies in the "third page" of $T \times I \times I$, forming Punc $S^{2} \times S^{1}$. 
Version II implies Version I, by Lemma I. To prove that Version I implies Version II, we introduce a third version.

Definition 2. A punctured ball sum is the disjoint union of a finite number of punctured balls. Each punctured ball may be punctured any finite number of times.

Version III of the theorem. Let $M$ be a 3-manifold embedded in $G \times I \times I$. If $b_{1}(M)=0$, then $M$ is a punctured ball sum.

Version I implies Version III, by examination of each component of $M$ individually. Version III implies Version II, since any punctured ball sum embeds in 3-space.

In our fourth and final version, we make a slight change in Version III; namely, in place of a graph $G$, we substitute a tree with only one vertex of high order.

Definition 3. For any positive integer $n$, an $n$-odd $Y$ is a tree with one vertex $v$ of order $n$, and $n$ other vertices of order 1 .

Version IV of the theorem. Let $M$ be a 3-manifold which embeds in $Y \times I \times I$, for some $n$-odd $Y$. If $b_{1}(M)=0$, then $M$ is a punctured ball sum.

Version III immediately implies Version IV, because every $n$-odd is a graph. Version IV implies Version III because any $G \times I \times I$ embeds in $Y \times I \times I$ for some $n$-odd $Y$. To establish this, we prove a simpler and stronger statement:

Lemma 2. For any graph $G$, there exists an $n$-odd $Y$ such that $G \times I$ embeds in $Y \times I$.

Proof. Let $N$ denote a regular neighborhood in $G$ of the vertices of $G$. We embed $N$ in $Y \times I$ so that each page of $Y \times I$ contains exactly one edge of $N$. The embedding of $N$ extends to an embedding of $G$ in $Y \times I$. This extension may be easily constructed on each arc of $G-N$ individually. Lastly, one extends this embedding of $G$ in $Y \times I$ to an embedding of $G \times I$ in $Y \times I$. This completes the proof of Lemma 2.

We thank the referee for suggesting Lemma 2.

The rest of this paper is devoted to a proof of Version IV of the Theorem.

\section{TRANSVERSE POSITION OF $M$ IN $Y \times I \times I$}

Regarding $Y \times I \times I$ as a "book with $n$ pages", we call the disk $B=v \times I \times I$ the binding, the $n$ components $P_{1}, P_{2}, \ldots, P_{n}$ of $Y \times I \times I-B$ are called the pages.

Definition 4. If $W$ is a subset of a 3-manifold $M$, we say that $W$ is neighborhood-bicollared in $M$ if a regular neighborhood of $W$ in $M$ is of the form $W \times[-1,1]$, with $W$ identified with the level $W \times 0$.

Observe that if $W$ is neighborhood-bicollared in $M$, then $W$ must be a 2-manifold. To see this, let $p$ be a point of $W$. The link of $p$ in $W$ must 
be connected, and has no vertex of order greater than 2 , because $W \times[-1,1]$ is a 3-manifold at the point $p \times 0$. Thus, this link is either an arc or a simple closed curve, depending if $p$ is a boundary point or interior point of $W$.

Definition 5. A 3-manifold $M$ in $Y \times I \times I$ is transverse to the binding $B$ if each component $W$ of $M \cdot B$ is neighborhood-bicollared in $M$ by the set $W \times[-1,1]$, and the two one-sided collars $W \times[-1,0)$ and $W \times(0,1]$ lie in two distinct pages $P_{i}$ and $P_{j}$ of $Y \times I \times I$.

If a 3-manifold $M$ is transverse to the binding in $Y \times I \times I$, then each component $W$ of $M \cdot B$ is an $n$-punctured disk in $B$, since $W$ is a connected 2-manifold subset of $B$. This fact is important, in that it provides us with an innermost curve of $b d M \cdot B$ on which we will later perform surgery.

Lemma 3. Any 3-manifold $M$ in $Y \times I \times I$ may be placed transverse to the binding. That is, if $M$ is embedded in $Y \times I \times I$, then there exists another embedding of $M$ in $Y \times I \times I$ which is transverse to the binding.

Proof. In one lower dimension, an easy example shows the difficulty and how to avoid it. Consider the subdisk $D$ of $T \times I$, for $T$ a triod, consisting of all of page 1 , the top half of page 2 , and the bottom half of page 3 . Then $D$ is a 2-manifold in $T \times I$ which is not transverse to the binding. The difficulty is that the midpoint $m$ of the binding is a "bad" point, in that any neighborhood of $m$ in $D$ is "3-paged" in $T \times I$. Note that $m$ is a boundary point on the disk $D$.

On the other hand, at any interior point, the disk $D$ is "2-paged." Our plan to avoid the difficulty caused by 3-paged points is to push $D$ into its own interior, thus making it locally "2-paged." The new disk may be placed in transverse position by resorting to the usual notion of general position in a 2-paged (Euclidean space) setting. Fortunately, this idea is valid in the higher dimensional situation as well:

Definition 6. A subset $X$ of $B \cdot M$ is called 2-paged if there are two integers $i$ and $j$ such that an open neighborhood of $X$ in $M$ is contained in $P_{i}+P_{j}+B$.

Every point $p$ of $B \cdot \operatorname{Int} M$ is 2-paged. To see this, suppose every open neighborhood of $p$ intersects the $i$ th page $P_{i}$. There exists an open neighborhood $N_{i}$ of $p$ in the "closed $i$ th page" $P_{i}+B$ such that $N_{i}$ lies entirely in Int $M$. The existence of three distinct such neighborhoods $N_{i}, N_{j}$, and $N_{k}$ would imply that $M$ must contain the set $T \times I \times I$, for $T$ a triod. Of course, no 3-manifold can contain such a set. In fact, this argument shows that the two integers $i$ and $j$ that we associate with the point $p$ are distinct; furthermore, $i$ and $j$ do not change if we move $p$ in the binding to any nearby point $q$. Thus, every component $C$ of $B \cdot \operatorname{Int} M$ is 2-paged in $Y \times I \times I$.

We now push $M$ into its own interior. That is, select any homeomorphic copy $M^{*}$ of $M$ such that $M^{*}$ lies in Int $M$. Let $C^{*}$ denote a component of $B \cdot M^{*}$. Since $C^{*}$ is a subset of a component $C$ of $B \cdot$ Int $M$, there exists 
a regular neighborhood $R^{*}$ of $C^{*}$ in $M^{*}$ such that $R^{*}$ lies in two pages of $Y \times I \times I$. That is, there exist two distinct integers $i$ and $j$ such that $R^{*}$ lies in $P_{i}+P_{j}+B$.

The 3-manifold $R^{*}$ is now moved into general position with respect to $B$ in the set $P_{i}+P_{j}+B$. Here, we regard $P_{i}+P_{j}+B$ as a cube in Euclidean 3-space, and we use the term "general position" in its usual sense in 3-space (no three points colinear, no four points coplanar). In general position, $R^{*}$ intersects $B$ transversally. Observe that this move of $R^{*}$ into general position with respect to $B$ may be entirely performed in a small neighborhood of $B$, so that it extends to an embedding of the 3-manifold $M^{*}$ in $Y \times I \times I$. The set $C^{*}$ has been replaced by transverse intersection of $M^{*}$ with $B$.

This procedure is now repeated for every component of $B \cdot M^{*}$.

This completes the proof of Lemma 3.

\section{Classification of surgeries as "Reducing" and "ENLARging"}

Our general plan is to reduce the number of simple closed curves in which $b d M$ intersects the binding $B$. This is accomplished by a sequence of "reducing surgeries". This reduction procedure finally yields a manifold $M^{\prime}$ which is disjoint from the binding. Each reducing surgery causes either $b_{0}(M)$ or $b_{2}(M)$ to increase by 1 , but $b_{1}(M)$ remains zero throughout. Thus, the fully reduced manifold $M_{r}$, which embeds in 3-space, must be a punctured ball sum. The inverse of reducing surgeries is called "enlarging surgeries". We show that each enlarging surgery preserves punctured ball sum, so we may retrace our steps from $M_{r}$ back to $M$, proving that $M$ is a punctured ball sum. Note that the manifold itself is not being reduced in any sense by this procedure. The complexity of placement of $M$ in $Y \times I \times I$, that is, the intersection of $b d M$ with $B$ in $Y \times I \times I$, is being reduced.

Definition 7. We define two types of reducing surgery.

2-handle addition: Let $D \times I$ be the product of a disk and an interval. Suppose that Int $D \times I$ is disjoint from $M$, and $b d D \times I$ lies in $b d M$. The reduced manifold $M^{\prime}$ is defined by $M^{\prime}=M+\operatorname{Int} D \times I$.

1-handle subtraction: Let $D \times I$ be the product of a disk and an interval. Suppose that Int $D \times I$ lies in Int $M$, and $b d D \times I$ lies in $b d M$. The reduced manifold $M^{\prime}$ is defined by $M^{\prime}=M-\operatorname{Int} D \times I$.

Lemma 4. Both types of reducing surgery preserve the property that the first betti number is zero. That is, if $b_{1}(M)=0$ before reducing surgery, then $b_{1}\left(M^{\prime}\right)=0$ after reducing surgery.

Proof. Adding a 2-handle to $M$ may be viewed homologically as attaching a disk $D$ to $M$ along $b d D$. This disk does not add nontrivial elements to the first homology group.

Since $b_{1}(M)=0$, any 1 -handle in $M$ separates $M$, so $b_{1}$ is also preserved under 1-handle subtraction. 
Definition 8. We define two types of enlarging surgery. They are the two inverse operations of the two reducing surgeries. In the notation of Definition 7 , they turn $M^{\prime}$ back into $M$.

2-handle subtraction: Let $D \times I$ be the product of a disk and an interval. Suppose that $D \times \operatorname{Int} I$ lies in Int $M^{\prime}$, and $D \times b d I$ lies in $b d M^{\prime}$. The enlarged manifold $M$ is defined by $M=M^{\prime}-D x \operatorname{Int} I$.

1-handle addition: Let $D \times I$ be the product of a disk and an interval. Suppose that $D \times \operatorname{Int} I$ is disjoint from $M^{\prime}$, and $D \times b d I$ lies in $b d M^{\prime}$. The enlarged manifold $M$ is defined by $M=M^{\prime}+\operatorname{Int} D \times I$.

Lemma 5. Let $M^{\prime}$ be a punctured ball sum. Let $M^{\prime}$ be altered by enlarging surgery, forming $M$. If $b_{1}(M)=0$, then $M$ is a punctured ball sum.

Proof. If $M$ is formed from $M^{\prime}$ by 2-handle subtraction, then the 2-handle lies in a component of $M^{\prime}$, a punctured ball $P$. Furthermore, this 2-handle connects two different components of $b d P$, because $b_{1}(M)=0$. Surgery changes $P$ to a punctured ball with one less puncture.

If $M$ is formed by 1 -handle addition, then the 1 -handle must connect two different components of $M^{\prime}$ as a boundary connected sum, because $b_{1}(M)=0$. The boundary connected sum of two punctured balls is a punctured ball. This completes the proof of Lemma 5.

\section{CONCLuSION}

We now assemble the various Lemmas into a proof.

Proof of Version IV of the theorem. Let $M$ be a 3-manifold which embeds in $Y \times I \times I$, for some $n$-odd $Y$. By Lemma 3, we place $M$ transverse to the binding in $Y \times I \times I$. Starting with the innermost simple closed curve of $b d M \cdot B$, we perform reducing surgery on $M$, which terminates with $M_{r}$, with $M_{r}$ disjoint from the binding. Since $b_{1}(M)=0$, Lemma 4 asserts that $b_{1}\left(M_{r}\right)=0$. Since $M_{r}$ is disjoint from the binding in $Y \times I \times I$, any component of $M_{r}$ is embedded in $I \times I \times I$. Furthermore, each component of $M_{r}$ has $b_{1}=0$. Lemma 1 tells us that each component is a punctured ball, so $M_{r}$ is a punctured ball sum. Lemma 5 guarantees that $M$ is a punctured ball sum. This completes the proof of the Theorem.

\section{REFERENCES}

1. D. B. A. Epstein, Embedding punctured manifolds, Proc. Amer. Math. Soc. 16 (1965), 175176.

2. D. Gillman and D. Rolfsen, The Zeeman Conjecture for standard spines is equivalent to the Poincaré Conjecture, Topology 22 (1983), 315-323.

3. E. Moise, Geometric topology in dimensions 2 and 3, Springer-Verlag, New York, 1977.

4. H. Seifert and W. Threlfall, A textbook of topology, Academic Press, New York, 1980.

5. E. C. Zeeman, On twisting spun knots, Trans. Amer. Math. Soc. 115 (1965), 471-495.

Department of Mathematics, University of California, los Angeles, California 90024-1766 